\title{
Advanced Automation and Robotics for High Volume Labour-Intensive Manufacturing
}

Gavin Lai, Nai Yeen; Jayasekara, Deepesh; Wong, Kok Hoong; Yu, Lih Jiun; Kang, Hooi Siang; Pawar, Kulwant; Zhu, Yingdan

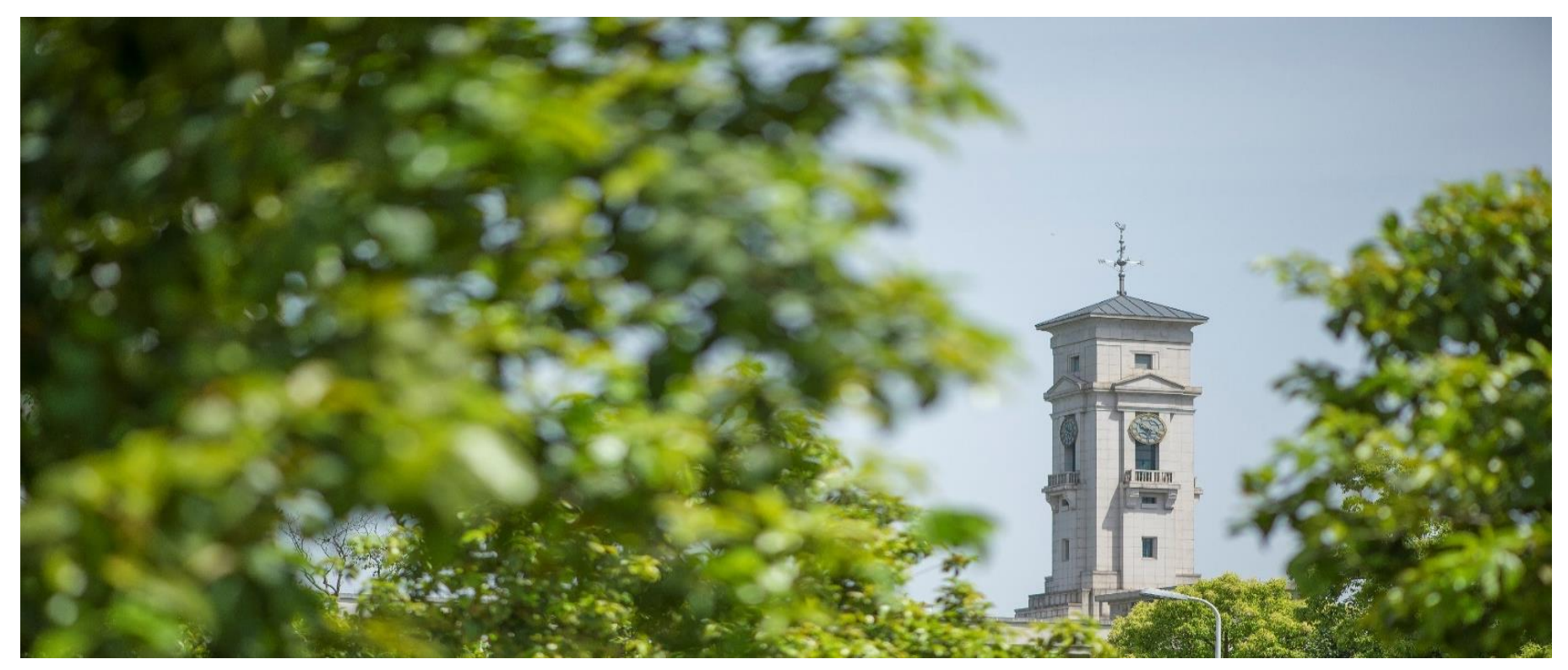


University of Nottingham Ningbo China, 199 Taikang East Road, Ningbo, 315100, Zhejiang, China.

First published 2020

This work is made available under the terms of the Creative Commons Attribution 4.0 International License:

http://creativecommons.org/licenses/by/4.0

The work is licenced to the University of Nottingham Ningbo China under the Global University Publication Licence:

https://www.nottingham.edu.cn/en/library/documents/researchsupport/global-university-publications-licence-2.0.pdf 


\title{
Advanced Automation and Robotics for High Volume Labour-Intensive Manufacturing
}

\author{
Nai Yeen Gavin Lai ${ }^{1}$, Deepesh Jayasekara ${ }^{1}$, Kok Hoong Wong ${ }^{1}$, Lih Jiun Yu$^{2}$, Hooi Siang Kang ${ }^{3}$, Kulwant Pawar ${ }^{4}$, \\ Yingdan $\mathrm{Zhu}^{5 *}$ \\ ${ }^{1}$ Department of Mechanical, Materials, and Manufacturing Engineering, University of Nottingham Ningbo China, \\ Ningbo, China, gavin.lai@nottingham.edu.cn \\ ${ }^{2}$ Faculty of Engineering, Technology \& Built Environment, UCSI University, Kuala Lumpur, Malaysia \\ ${ }^{3}$ School of Mechanical Engineering, Universiti Teknologi Malaysia, Johor Bahru, Malaysia \\ ${ }^{4}$ Division of Operations Management \& Information Systems, University of Nottingham, Nottingham, United Kingdom \\ ${ }^{5}$ Zhejiang Provincial Key Laboratory of Robotics and Intelligent Manufacturing Equipment Technology, Ningbo Institute \\ of Material Technology and Engineering, Chinese Academy of Sciences, Ningbo, China, y.zhu@ nimte.ac.cn
}

\begin{abstract}
High volume, labour-intensive manufacturing operations pose several challenges for successful operations. It is an even bigger challenge to move away from manual dependency and shift towards automation of production processes. There seems to be no general agreement among researchers on what are the critical success factors (CSFs) on the implementation of advanced automation and robotics processes in the manufacturing environment. Success factors vary across the manufacturing industry and also organizational units. This paper attempts to elicit the opinion and perception of academic professionals with links to high volume labour-intensive manufacturing industry. A systematic literature search was conducted on the topic and semi-structured interviews were conducted with experts to gather their views on CSFs. Results from the literature search and interviews revealed that factors related to Organization Strategy, Culture, and Structure; Top management support; Past experience, staffing, and learning; Project management, planning, and control; Finance, and resource availability; Technology maturity and vendor support; to be critical for the success of the production process automation project initiatives. Findings from this study can be used as guidelines to ascertain CSFs for organizations and strategy planning. Manufacturing organizations can also use this study to evaluate their organizational readiness and enhance the level of automation further.
\end{abstract}

Keywords-Critical success factors, Automation and robotics, Manufacturing, Industry 4.0

\section{INTRODUCTION}

Industrial automation has evolved through many phases from automation in single production lines, to full-scale computer integrated manufacturing systems over the past decades by replacing much of labour-intensive manual processes and increasing the level of automation in many industries [1]. New digital technologies such as the Internet of things, cyber-physical systems (CPS), and novel concepts such as the factory of the future, Industry 4.0 and cloud manufacturing (CMfg) have fuelled the hunger for further automation and maintain competitiveness in the manufacturing sector [2]. Moreover, Industrial robotics, in the past 50 years, have significantly transformed the manufacturing industries around the world by replacing humans in various repetitive, difficult, or dangerous tasks and at present collaborative human-robot working environments are becoming increasingly popular due to enhanced flexibility and efficiency [3]. It is further evident in the literature that these advanced manufacturing systems which enabled an increased level of automation in the shop floor have paved the way to achieving the goal of TQCSEFK (i.e. fastest time-to-market, highest quality, lowest cost, best service, greenest environment, maximum flexibility, and highest knowledge) for modern manufacturing enterprises $[4,5]$ and therefore, embracing automation is inevitable at present to survive in this fiercely competitive market.

However, automation is a complex process and according to Strmčnik and Černetič [6], it belongs to the class of most complex, demanding, and challenging human-machine systems. Therefore, careful attention is required to properly balance the human-machine relationship in all phases of design, implementation, and operation. When it comes to high volume, labour-intensive manufacturing industries, this is even more challenging. Labour-intensive 
manufacturing plays a major role in many developing countries even at present. For example, China which is undoubtedly the manufacturing powerhouse in the world is still considered as a country that is at the forefront of labour-intensive manufacturing [7]. Further, a significant portion of the materials required for many labourintensive manufacturing industries in developing countries such as Malaysia, Indonesia, and Sri Lanka are supplied by China i.e. apparel, footwear, toys, furniture, etc. These countries all together cater the largest portion of the worldly demand. Hence, automation and robotics play a significant role in assuring the sustainability of these industries in the future. The adverse effects of lack of automation are very much evident, especially during the COVID-19 pandemic. Many industries in developing countries were stranded due to their high dependency on labour, lack of supply, and disrupted supply chains and authors believe the impact from such a chaotic worldly event could have been minimized if these industries were automated but, unfortunately, it was not the case.

Therefore, this research was conducted to identify critical success factors (CSFs) which enable increased utilization of automation and robotization in high volume, labour-intensive manufacturing in developing countries. An extensive literature search was conducted by using research databases such as Scopus, Science Direct, and Google Scholar to identify relevant past studies relating to CSFs. Analysis of CSFs and the valuable insights from experienced professionals in numerous manufacturing industries from China, Malaysia, and Sri Lanka were taken into account when answering the following research questions "Which CSFs must be considered to ensure a successful implementation of automation and robotics?' and 'What are the opinion and perception of academic experts involved with the automation and robotics implementation in manufacturing settings?'

The authors thoroughly believe the findings of this study provide valuable guidance to any high-volume, labourintensive manufacturing industry to develop proper strategies and swiftly transform their manual processes into efficient automated processes.

\section{THEORETICAL BACKGROUND/LITERATURE REVIEW}

\section{A. Advance automation, robotics, and AMT in manufacturing}

Automation is often seen as the go-to solution to achieve cost-efficient high-volume manufacturing and alleviate humans from repetitive, heavy, or dangerous tasks. However, research has shown that increasing levels of automation in unforeseen production situations may lead to production disturbances [8]. Therefore, when implementing robotics and automation solutions in high volume, labour-intensive manufacturing industries, a very important aspect is to understand the precise level of automation required to maximize the throughput while taking steps to improve the efficiency of labour-oriented processes [9]. The importance of finding appropriate levels of automation has been emphasised by many authors [10, 11]. A concept closely related to the implementation of automation and robotics in manufacturing is Advanced Manufacturing Technologies (AMT) which is defined as "an automated production system of people, machines, and tools for the planning and control of the production process, including the procurement of raw materials, parts, and components, and the shipment and service of finished products" [12]. Studies on AMT adoption is closely linked to that of automation projects in industry settings [13].

Even though there is a surge in enthusiasm towards smart factories, factories of the future, cloud manufacturing (CMfg) and Industry 4.0 at present, most of the sophisticated manufacturing systems available today are not fully autonomous, rather, the production systems are semi-automated and humans still play a vital role in tackling unforeseen scenarios. Therefore, both advanced manufacturing systems and skilled workers are equally important to achieve flexible and efficient production [11]. Nevertheless, increased demand in customization, time-to-market, and product complexities to match consumer requirements, have led to increasingly complex production systems. Adding to that, ever-increasing wage rates in developing countries and competition in the market, have compelled manufacturers to increase their level of automation every year.

The transformation of manual processes to fully automated manufacturing processes cannot be done feasibly in one step. Parasuraman, et al. [10], describe automation as a continuum of levels, from fully manual processes performed only by human operators, followed by intermediary levels where tasks are performed by both human 
and machines (or computers), to the highest level of full automation performed only by machines (or computers). After analysing different taxonomies presented by many authors for levels of automation (LOA) discussed in the review paper by Vagia, et al. [14], taxonomy presented by Endsley and Kaber (1999) was identified as a suitable taxonomy for the purpose of this study, which comprises 10 levels of automation as shown in Table 1.

TABLE 1: LEVELS OF AUTOMATION BY ENDSLEY AND KABER (1999) CITED IN VAGIA, ET AL. [14].

\begin{tabular}{|c|c|c|}
\hline Levels & Description & Description \\
\hline Level 1 & Manual operation & The human performs all tasks \\
\hline Level 2 & Action support & $\begin{array}{l}\text { The human still holds the main role and the system assists with the performance } \\
\text { of the selected action. }\end{array}$ \\
\hline Level 3 & Batch processing & $\begin{array}{l}\text { Human generates and selects the options to be performed, which are later on } \\
\text { turned over to the system to be carried out automatically. }\end{array}$ \\
\hline Level 4 & Shared control & $\begin{array}{l}\text { Both the human and the computer generate possible decision options and the } \\
\text { tasks are shared between the human and the system but the human retains full } \\
\text { control over the selection of options. }\end{array}$ \\
\hline Level 5 & Decision support & $\begin{array}{l}\text { The computer generates a list of decision options, which the human can select } \\
\text { from. The operator may generate options. Once the human has selected an } \\
\text { option, implementation is done by the computer. }\end{array}$ \\
\hline Level 6 & Blended decision making & $\begin{array}{l}\text { The computer generates a list of decisions and selects the most appropriate ones } \\
\text { but the task is carried out only after human consent. The human operator can } \\
\text { select other options if required. }\end{array}$ \\
\hline Level 7 & Rigid system & $\begin{array}{l}\text { The system presents only a limited set of actions to the operator. The operator } \\
\text { has little discretion on the options. }\end{array}$ \\
\hline Level 8 & $\begin{array}{l}\text { Automated decision } \\
\text { making }\end{array}$ & $\begin{array}{l}\text { The system selects the best option to implement and carries out the tasks based } \\
\text { upon a list of alternatives it generates. }\end{array}$ \\
\hline Level 9 & Supervisory control & $\begin{array}{l}\text { The system generates options, selects the options, and carries out the task. The } \\
\text { human intervenes only if necessary. }\end{array}$ \\
\hline $\begin{array}{c}\text { Level } \\
10\end{array}$ & Fully autonomous & The system carries out all actions. \\
\hline
\end{tabular}

\section{B. Critical Success Factors (CSFs)}

The beginning of the study on critical success factors (CSFs) can be traced back to 60 years ago with the work of Daniel in 1961 from the field of management who had discussed "success factors" relating to industry [15]. Rockart who is one of the key pioneers on the subject of CSFs considers CSFs to be the limited number of areas (factors) in which if they are adequate could ensure successful competitive performance for a company or organization [16]. In other words, “....few key areas, things must go right for the business to flourish" [16]. Therefore the understanding of CSFs is an important topic for managers and organizations if they seek to ensure competitive performance of their unit. However, it is important to stress that CSFs are unique to a particular situation and are not a standard set of "key indicators" [16]. The study of CSFs may have started from the perspective of top management level but CSFs have now been studied at different levels and across different industries or settings. Past studies have indicated an established link between CSFs and organization performance [17]. Furthermore, CSFs can be either tactical or strategic [18]. In many cases, a combination of both tactical and strategic CSF is required to ensure overall success [19]. Strategic CSFs are dominant in the initial phase of a project or implementation while gradually the shift will be towards the use of tactical CSFs during the operational phase. The study on CSFs has been acknowledged to be effective in improving the effectiveness of project implementation although there is yet to be an all-encompassing list of factors agreed by all researchers that influence project success $[20,21]$ 
CSFs can be considered either at the industry level or alternatively be viewed at the organization or company level [22]. Studies on CSFs have been implemented in a variety of disciplines including information systems, project management, construction, manufacturing processes, business improvement, and technology implementation. Specifically, there have been numerous studies on the topic of CSFs relating to the implementation of automation, robotics, and advanced manufacturing technology AMT (see e.g. [1, 13, 23-26]).

\section{METHODOLOGY}

In order to extract the in-depth views of experts on CSFs for automation and robotics in high volume labour dependent manufacturing industries, a qualitative approach was employed. First, a systematic literature search was carried out by the authors that resulted in the identification of a number of past studies that are highly related to the CSFs of automation and robotics project implementation in a manufacturing setting. These are highly related studies on success factors and CSFs (see [1, 13, 23-26]). The findings provided the basis for the formulation of the guide questions for the semi-structured interview of key professionals of the industry. To refine the questions, a review discussion on the questions was initiated by the authors which enable some modifications to the initial questions. Some items were merged together as they were along the same direction, while repetitive items as well as irrelevance items to the targeted area of study were deleted. Additionally, the terminology was also checked to ensure that they matched the norm of the targeted scope of the study. A final list of 12 guide questions was determined after these rounds of reviews and was used for the semi-structured interview process.

The interviews were purposefully selected, with invitations extended to experts who are active in the industry, teaching and research on automation and robotics. A total of 15 relevant expert professionals were invited to participate in the interviews. A covering letter explaining the purpose of the study was sent to each participant and participation was voluntary and anonymous. The invited participants are key academics with industry knowledge and exposure in the field of automation and robotics. Apart from actively conducting research in the field of automation and robotics, these experts also had been involved with industries using automation and robotics either from past working experience or industrial consultancy assignments.

As all of them accepted the invitation, a total of 15 semi-structured interviews were successfully conducted with the experts. This enables the study to gather essential personal insights, opinions, and experiences of the experts regarding critical success factors in the automation and robotics of production lines. These valuable inputs from the experts were helpful to validate and affirmed the findings of the initial literature search.

The thematic analysis approach was used to analyze the interview outcomes. The thematic analysis approach is widely used in qualitative research and enables the examining themes or patterns of meaning within the collected detailed textual data [27]. Further details on the thematic analysis procedure are well presented in [27]. All the recordings of the interview sessions were transcribed into text format. Next, a member of the research team had to compare the transcript with the audio recording to ensure the correctness of the transcription process before proceeding with the analysis stage. All researchers had also read through the transcribed interviews for accuracy and raised any possible issues. Subsequently, each of the authors independently open coded the interviews to reveal emerging themes and remarking anything that they believe to be related to the main research questions. The research team next compared the notes, discussed, and adjusted the individual person coding for consistency. The datasets were then next examined iteratively, enabling emerging themes to be developed further. Any linkages in the text and themes were also openly discussed and debated among the researcher until a consensus is reached.

\section{Guide Questions}

1. What is/was your involvement in the automation of manufacturing processes?

2. How many years of industry/consulting experience do you have with automation or robotics in manufacturing in total? 
3. How do you rate the level of automation in the industry that you are/were involved with? (i.e. labour intensive, semi-automated, fully autonomous)

4. What impact does advance automation \& robotics implementation have on the manufacturing company/industry strategy, culture, and structure?

5. How much prior experience the industry or company had with automation and robotics prior to the implementation in the manufacturing processes?

6. Were changes required in the staffing strategy of the industry or company to successfully implement automation of the manufacturing processes? How important is training for staff to prepare them for automation \& robotics in manufacturing processes?

7. How important is the readiness (or maturity) of the technology to ensure a successful implementation and operation thereon? (i.e. off-the-shelf solutions vs customized solutions vs tailor-made solutions)

8. How essential is the support from the automation technology supplier (or vendor) to successfully implement automated solutions in the manufacturing processes?

9. How important is the availability of the top management support to successfully implement advanced automation solutions in the manufacturing processes?

10. How important is the availability of required financial resources for the implementation of advanced automation and robotics in the manufacturing processes?

11. How important are project planning, management, and control when deploying automation and robotics processes in the manufacturing environment?

12. Are there any other comments that you would like to make regarding the successful advance automation and robotics' implementation in manufacturing processes?

\section{RESULTS AND DISCUSSIONS}

The selection of interview participants was contacted through the personal networks of authors in the respective countries. The potential experts were known to the authors to be active in the areas of automation and robotics relating to manufacturing, and there were questions in the interview sessions that relate to this profile. All of the experts who were approached had accepted the invitation to participate. A review of the questions on experience and involvement further confirms the initial choice of the selected interview participant by the authors as they are all experienced persons with high familiarity on the topic.

All of the experts had been involved with automation and robotics environment of Level 2-9 based on the taxonomy presented by Endsley and Kaber (1999) Vagia, et al. [14]. In other words, the automation was used together with manual labor in the industry or companies that the experts were involved with.

The analysis of the inputs from the experts reveals several interesting aspects of the implementation of automation and robotics in manufacturing processes in labour-intensive industry. All of the experts' inputs were first reviewed for applicability and later group into different themes. The study indicates that there are six key themes of critical success factors that are vital towards the implementation of automation and robotics projects or initiatives in high volume labour-intensive manufacturing industries.

\section{A. Organization Strategy, Culture, and Structure}

Most experts that contributed to this study agreed that change to the organization is inevitable with the introduction of automation and robotics in manufacturing processes. How the organization and its staff react towards the change has a key impact on the success of the implementation effort. The company may need to implement changes to the organization structure to harness the maximum benefits from the implementation of advanced automation technologies $[22,28,29]$. On the other hand, the compatibility of the technology with current organization design is a key success factor for automation project implementation [30]. The large degree of change affecting the organization from the implementation project would likely drive up staff fear and resistance. Past research has indicated that organizations looking to implementing automation and robotics successfully may need to redesign their organizational structure and organizational processes [31]. 
Additionally, the existing structure of the organization may affect the implementation of advanced manufacturing technology automation [32]. The staff's expectation of the technology and the staff's fear of the technology displacing them in the new structure must be adequately addressed. Moving towards the right organizational structure would help a company be more successful in implementing automation and robotics in manufacturing settings [33, 34]. However, full staff involvement, understanding, and support will also be critical for success.

For long-establish organizations, existing or legacy company structure may not always be suitable for the implementation of automated and robotics-based manufacturing settings. Boyer et al. had noted how reshaping the company structure may potentially enable a smoother implementation of advanced automation for production [35]. Implementing new advanced manufacturing technologies while failing to review and reorganize the company structure leads to fewer benefits from the implementation [31]. The introduction of advanced automation technologies in manufacturing could be successful if the existing organization structure could accommodate the required changes. An existing company manufacturing strategy and initiatives can also be complementary to that of advanced manufacturing technology and automation and initiatives such as those envisioned in Industry 4.0 [36], but proper review and consideration is essential.

The experts have also commented on the potential shift of company culture in terms of productivity and efficiency. If the implementation of automation of manufacturing is seen as a manufacturing best practice, its success would be highly dependent on the organization culture driving the implementation [37]. The impact of organizational culture on the implementation of advanced manufacturing technology is evident [38]. Companies with a more flexible organization culture adaptive to changes will be more successful in the implementation of automation and robotics [25]. A number of the interviewees also indicated that if automation and robotics implementation is part of the organization's vision and strategy, implementation success would be much higher. This is in line with past research which acknowledges that the company strategy influences the successful implementation of automation and robotics in manufacturing [39-41]. It is expected that organization strategy or more precisely, the manufacturing strategy will have a direct influence on the success of automation and robotics implementation in production.

\section{B. Top management Support}

Literature had indicated that top management support (TMS) and attitude towards advance manufacturing technology automation plays a key role in ensuring the success of its implementation [42-46] and the findings of this study reinforce that notion. All of the contributors to this study had stressed the importance of TMS in ensuring the successful implementation of automation and robotics in a manufacturing environment. Furthermore, TMS is considered to be the most important critical success factor for project or initiative success and is not just one of many factors. [47].

All of these experts agreed that without TMS, implementation of automation and robotics in manufacturing would likely fail. Some had commented that top management of the organization must have a good understanding of automation and robotics implementation and must be able to link its importance to the organization strategy as well. Top management provides leadership either directly or through delegation of authority to ensure the implementation of automation or robotics project could progress smoothly and received the required cooperation from the whole organization. TMS could help boost employee morale and provide influence or leverage to the change implementation $[48,49]$ brought upon by the introduction of automation. The availability of support would lead to wider participation and support from all stakeholders in the company or organization to ensure the success of the implementation.

Top management should not only be considered as the provider of financial resources but also as the authority to be able to influence the organization, gather further support, provide strategic direction and further directives needed for the implementation of transformative automation and robotics project in manufacturing. Top management commitment in supporting automation and robotics implementation in manufacturing provides 
leadership and direction for the whole organization staff to support the implementation effort. Organizational members or staff resistance is a top reason for change implementation projects failure [50]. Apart from changes in the organization structure and culture from the implementation of advanced automation in manufacturing, management must seek to build up mutual trust and cooperation in the organization to ensure success [51].

\section{Past Experience, Staffing, and Learning}

The introduction of automation and robotics creates a significant change to an organization, likely changing the working environment for many of the staff there. The level of change from automation implementation will depend on the company's prior experience (and its staff) with automation and robotics. Prior or past experiences with the usage of automated processes and robotics will enable an easier learning curve for the organization in the implementation of more advanced solutions. The experts consulted agreed with the idea that a gradual change in the introduction of automated solutions will be a better approach to ensure success. Changes in an organization, including that of its manufacturing process and strategy, should be a gradual shift and takes into account the level of organizational learning that occurs [52]. Prior experience with simpler forms of technology may help the implementing organization to gather relevant knowledge and experience which will gradually enable it to move on to more complex generations of automation solutions [53]. Some of the existing manual tasks and responsibilities will be displaced and replaced by automation processes. The implementation of automation and robotics in manufacturing would require more skilled workers who should be entrusted to manage and operate the associated equipment or resources [54].

Staff with new skills and knowledge are needed by the implementing organization where the following approaches may be taken to address this. The organization could upskill its existing workers to the level needed for the operations of automation solution and also change the organization staffing strategy where staffs with a higher education and skill level are recruited into the organization. The likelihood as agreed by the experts that participated in this study is that the combination of both higher educated staff and manual labour staff will still be required for the organization as the journey to full automation of manufacturing is a long and iterative process. Even in a fully automated process, there could still be a need for manual labour intervention in some supporting and auxiliary roles.

Organizations will also need to invest in training and continuous development of their workforce to harness the maximum benefit of the automation of production. Education and training is an important asset for manufacturing organization and has been proven to enhance the performance over firms in complex manufacturing environment $[55,56]$ which is evident with the introduction of automation and robotics-based manufacturing process. Moreover, staff training and education for new technology implementation are positively associated with productivity performance [57]. Without the proper support and training for the workers, introducing new manufacturing technology will be a challenging affair [58]. Past research has indicated that training is an important aspect of the introduction of advanced manufacturing technology $[59,60]$.

\section{Project management, planning, and control}

The experts interviewed also shared the importance of having proper planning and project management in place for the implementation of automation and robotics in manufacturing. It has been noted that proper planning is an important requirement for the success of advance manufacturing technology implementation [61]. Implementing a more advanced level of automation and robotics in a manufacturing environment will likely need to be carried out as a series of projects with proper planning and control in order to ensure success. For larger organizations and companies, this is well-rooted in the company structure and culture; however, it may be a big challenge for smaller organizations due to the initiative's scale and complexities.

Selecting the right people and forming the right project team was also mentioned as a key part of a successful initiative. The presence of a project "champion" could also help boost the performance of the project implementation [13, 62]. The experts sharing their experiences had indicated that project teams with members from different disciplines and stakeholders in the organization could smoothen the implementation project. However, 
this again requires a strong "champion" for the team and TMS to be truly effective. Some organizations would have been accustomed to the scale and complexities involved with the implementation project, while the smaller organization would consider the implementation project to be very challenging. The project management, planning, and control setup in a larger organization are more established than that of SMEs [63]. Proper project management, planning, and control enable the delivery of the expected outcomes and within the required budget and time. Experts also agreed that the success of the implementation of automation in manufacturing is not guaranteed by the availability of resources allocated to it, but by the proper management and control of the available resources.

\section{E. Finance and resources availability}

The response from the experts covered by this study indicates that financial matters are a key determinant in the outcomes of automation and robotics implementation. All of the experts had the opinion that automation will require sufficient funding and the cost will not be a nominal sum for most of the organizations involved. It has been demonstrated that cash flow strength has a profound effect on the decision to upgrade manufacturing technologies [64]. Larger organizations will fare better as they have a better ability to plan and to source for the required funds to implement automation and robotics initiatives. However, no matter the size of the company, planning in advance for the initiative and the budget required is key. In advanced technology manufacturing investment, proper planning and financial analysis could ensure the success of the initiative [65].

Most of the respondents had touched on the financial side of resources needed for successful implementation; however, the physical and non-financial resources such as available space, expertise, experience, management know-how, planning, and human resources are also essential. These and more are covered by the other themes identified in this study. Conventional financial techniques may not be adequate to effectively analyze the viability and benefits of advanced manufacturing automation projects [66]. Additionally, it was noted from the interview sessions that proper provision of financial resources should focus not only on acquiring the physical hardware and equipment but also for the proper operation and maintenance as well. Some companies implemented automation and robotics as a series of small projects, while others prefer a transformative large scale project. In the latter case, seeking the right and sufficient financial resources is even more essential. The involvement of the finance department or personnel in justification activities will have a positive impact on overall advanced manufacturing automation project implementation performance [67].

Some experts had also suggested the use of cost-benefit analyses, return on investment, and other accounting tools in organizations or industries to help plan for automation project implementation. Prior to the implementation of the automation project, this information could serve as an important tool to obtain buy-in and support from top management for the project implementation. After implementation, the performance of the automation implementation can still be systematically tracked and reported for management consideration. There are many past studies which had focused on specific tangible measures of advance manufacturing technology including automation implementation benefits from a financial performance perspective [38]. These serve as a good reference for future project consideration.

\section{F. Technology maturity and vendors support}

With the advancement of new technologies, modern-day manufacturing automation is focused on the possibility of end-to-end horizontal integration of processes utilizing digital technologies such as cyber-physical systems, machine-to-machine communication, internet of things, cloud computing, and wireless sensor networks to attain a higher level of automation, flexibility, and efficiency in production floor or even across multiple distributed manufacturing plants. According to an expert, considering the latest developments in smart digital technologies, Industries are willing to participate in implementing low readiness technologies, provided there is a strong potential for financial return. CPS and CMfg are considered key enablers of the fourth industrial revolution (Industry 4.0) [68]. However, according to Leitão, et al. [69], there are many challenges faced during the application of industrial automation using new complex technologies such as CPS at present (Table 2), and similarly, CMfg is a new 
manufacturing paradigm which has not reached maturity [70]. Therefore, increased maturity of technology plays an important role in the successful utilization of digital manufacturing automation.

TABLE 2: KEY CHALLENGES IN INDUSTRIAL CPS, ADAPTED FROM LEITÃO, ET AL. [69].

\begin{tabular}{|l|l|l|}
\hline Area & Key Challenges & Maturity \\
\hline CPS Capabilities & Real-time control of CPS systems & $4-7$ years \\
& Real-time CPS SoS (System of Systems) & $3-5$ years \\
& Optimization in CPS and their application & $4-7$ years \\
& On-CPS advanced analytics & $3-5$ years \\
& Modularization and servification of CPS & $3-5$ years \\
& Energy-efficient CPS & $3-5$ years \\
\hline CPS Management & Lifecycle management of CPS & $5-8$ years \\
& Management of (very) large scale CPS and CPS-SoS & $5-8$ years \\
& Security and trust management for heterogeneous CPS & $5-8$ years \\
\hline CPS Engineering & Safe programming and validation of CPS SoS & $5-10+$ years \\
& Resilient risk-mitigating CPS & $5-10+$ years \\
& Methods and tools for CPS lifecycle support & $3-7$ years \\
& New operating systems and programming languages for CPS and CPS- & $3-6$ years \\
& SoS & $3-6$ years \\
& Simulation of CPS and CPS-SoS & \\
\hline CPS Infrastructures & Interoperable CPS services & $2-5$ years \\
& Migration solutions to emerging CPS infrastructures & $3-6$ years \\
& Integration of heterogeneous/mobile hardware and software technologies & $2-4$ years \\
& in CPS & $3-5$ years \\
& Provision of ubiquitous CPS services & $3-6$ years \\
& Economic impact of CPS Infrastructure & \\
\hline CPS Ecosystems & Autonomic and self CPS & $7-10+$ years \\
& Emergent behaviour of CPS & $7-10+$ years \\
& CPS with humans in the loop & $2-5$ years \\
& Collaborative CPS & $5-8$ years \\
\hline CPS & Information & Artificial intelligence in CPS \\
Systems & Cross-domain large-scale information integration to CPS infrastructures & $7-10+$ years \\
& Transformation of CPS data and information analytics to actionable & $4-8$ years \\
& knowledge & $6-10+$ years \\
& Knowledge-driven decision making/management & \\
\hline
\end{tabular}

This was agreed by many of the experts in this study as they believe that priority should be given to mature technologies as high volume manufacturing companies cannot afford to have failures. It was also emphasized that failed automation due to lack of readiness might even lead to a total production stop situation that negatively impacts the profitability of the company. Therefore, understanding the readiness of innovative manufacturing technologies is crucial for industrial decision-making [71]. However, on some occasions, mature technologies might not be able to meet industry requirements. This was highlighted by an expert saying new products that are manufactured often need new technologies, and therefore, staff members from the organization will be working closely with equipment technology vendors in most cases years in advance to develop the right technology that meets the production needs. Nevertheless, many experts agreed that off-the-shelf solutions or customized solutions with mature technology would benefit the organization since they can be operationalized in a short period of time compared to tailor-made or in-house developed solutions using new technologies. It is also important to note here that not only the maturity of technology but also the organizations' technological readiness play an important role in successfully adopting modern technologies $[70,72]$.

The successful deployment of complex systems will not be possible without the support from vendors. Vendor support was highlighted as one of the key success factors in implementing ERP systems in many literature[73] and many experts agreed on this. The reason was highlighted by an expert in this manner "we need support from the 
supplier mainly because these automation technologies are relatively new for many industries and we need support to grasp the knowledge and to get machine operationalized as soon as possible. Machines usually come with detailed documentation like manuals but still without proper commissioning support, since we don't have enough experience in automation, it can become very challenging". Therefore, it is evident in literature and verified by the experts that technology readiness (or maturity) and vendor support is a critical success factor for successfully implementing robotics and automation in high volume labour-intensive manufacturing industries at present and in the future.

\section{G. Summary}

The experts who participated in this study provided an insightful account and opinion of the CSFs related to the implementation of advanced automation and robotics in a manufacturing environment. CSFs identified using an extensive literature search in this study agreed with the key inputs given by experts during the interviews. The CSFs were grouped in the following themes, Organization Strategy, Culture, and Structure; Top management support; Past experience, staffing, and learning; Project management, planning and control, Finance, and resource availability; Technology maturity and vendor support. Notably, all of the experts had felt strongly on Top management support as the most influential factor in their view but were also quick to add that ultimate success in implementation would still not be possible if the rest of the other factors were neglected. It is evident from the analysis that CSFs are interrelated, and discussion in the proposed themes could enable a better understanding of what would make production process automation project initiatives successful. Additionally, as the world moves towards high-performance manufacturing in the era of Industry 4.0, it is essential to note that automation and robotics technological factors alone will not be sufficient to ensure efficiency and competitiveness. Understanding the interplay of a host of factors will be essential.

\section{CONCLUSION}

This study had explored the CSFs of implementing automation and robotics in a high volume labour-intensive manufacturing environment. Using a mix of literature search and interviews with key experts related to the topic of study, this study had presented six key themes of CSFs relevant to the successful implementation of automation and robotics in a high volume labour-intensive manufacturing environment. The experts that participated in the interviews had all agreed with the initial findings from the literature search on what contributes to successful implementation outcomes. Although the experts agree that all of the areas brought up are critical to success, some factors are considered by them to be more important than others. The top success factor emphasized by them is top management support, which is in agreement with the literature on CSFs. It was also shown that the implementation of automation and robotics in a manufacturing environment is a complex task, and success cannot be guaranteed by a single factor or two. Instead, multiple factors need to be considered, and often, the factors are interrelated, as shown by the six CSFs themes presented in this study. Findings from this study will be helpful for academics and industrial practitioners when discussing CSFs and the planning of manufacturing organization strategies relating to automation and robotics. There is a recent resurgence of interest on the use of automation and robotics, as discussed in the many literature relating to Industry 4.0, Cloud Manufacturing, Industrial Internet of things, and much more. Further research on the CSFs of automation and robotics implementation will definitely be required in an ever-changing digital manufacturing era.

\section{REFERENCES}

[1] H. Takanaka, "Critical success factors in factory automation," Long Range Planning, vol. 24, pp. 29-35, 1991/08/01/ 1991.

[2] C. Biegler, A. Steinwender, A. Sala, W. Sihn, and V. Rocchi, "Adoption of Factory of the Future Technologies," in 2018 IEEE International Conference on Engineering, Technology and Innovation (ICE/ITMC), 2018, pp. 1-8. 
[3] S. Robla-Gómez, V. M. Becerra, J. R. Llata, E. González-Sarabia, C. Torre-Ferrero, and J. Pérez-Oria, "Working Together: A Review on Safe Human-Robot Collaboration in Industrial Environments," IEEE Access, vol. 5, pp. 26754-26773, 2017.

[4] F. Tao, L. Zhang, V. C. Venkatesh, Y. Luo, and Y. Cheng, "Cloud manufacturing: a computing and serviceoriented manufacturing model," Proceedings of the Institution of Mechanical Engineers, Part B: Journal of Engineering Manufacture, vol. 225, pp. 1969-1976, 2011/10/01 2011.

[5] Y. Zhang and F. Tao, "Chapter 1 - Introduction," in Optimization of Manufacturing Systems Using the Internet of Things, Y. Zhang and F. Tao, Eds., ed: Academic Press, 2017, pp. 1-20.

[6] S. Strmčnik and J. Černetič, "The Time Dimension of Critical Automation Success Factors," IFAC Proceedings Volumes, vol. 34, pp. 115-119, 2001/09/01/ 2001.

[7] P.-C. Chang and Y.-K. Lin, "Fuzzy-based system reliability of a labour-intensive manufacturing network with repair," International Journal of Production Research, vol. 53, pp. 1980-1995, 2015/04/03 2015.

[8] J. Frohm, "Levels of automation in production systems," Department of Product and Production Development Production Systems, Chalmers University of Technology, Göteborg, Sweden, 2008.

[9] V. Lindström and M. Winroth, "Choosing Levels of Automation in Production Systems: Finding Critical and Supportive Factors," in Proceedings of the 12th International EurOMA Conference on Operations and Global Competitiveness, Budapest, Hungary, 2005, pp. 1593-1601.

[10] R. Parasuraman, T. B. Sheridan, and C. D. Wickens, "A model for types and levels of human interaction with automation," IEEE Transactions on Systems, Man, and Cybernetics - Part A: Systems and Humans, vol. 30, pp. 286-297, 2000.

[11] J. Frohm, V. Lindström, M. Winroth, and J. Stahre, "The Industry View on Automation in Manufacturing," IFAC Proceedings Volumes, vol. 39, pp. 453-458, 2006/01/01/ 2006.

[12] J. M. Pennings, "Information technology in production organizations," International Studies of Management \& Organization, vol. 17, pp. 68-89, 1987.

[13] H. Zhao and H. C. Co, "Adoption and implementation of advanced manufacturing technology in Singapore," International Journal of Production Economics, vol. 48, pp. 7-19, 1997/01/10/ 1997.

[14] M. Vagia, A. A. Transeth, and S. A. Fjerdingen, "A literature review on the levels of automation during the years. What are the different taxonomies that have been proposed?," Applied Ergonomics, vol. 53, pp. 190202, 2016/03/01/ 2016.

[15] D. R. Daniel, "Management information crisis," Harvard business review, pp. 111-121, 1961.

[16] J. F. Rockart, "Chief executives define their own data needs," Harvard business review, vol. 57, pp. 81-93, 1979.

[17] D. Chrusciel and D. W. Field, "From critical success factors into criteria for performance excellence-An organizational change strategy," Journal of industrial technology, vol. 19, pp. 1-11, 2003.

[18] C. P. Holland, B. Light, and N. Gibson, "A critical success factors model for enterprise resource planning implementation," in Proceedings of the 7th European conference on information systems, 1999, pp. 273-287.

[19] B. Ward, "Planning for profit," Chapter, vol. 5, pp. 103-146, 1990.

[20] A. P. Chan, D. Scott, and A. P. Chan, "Factors affecting the success of a construction project," Journal of construction engineering and management, vol. 130, pp. 153-155, 2004.

[21] J. Wateridge, "IT projects: a basis for success," International journal of project management, vol. 13, pp. 169$172,1995$.

[22] L. Raymond, "Operations management and advanced manufacturing technologies in SMEs," Journal of Manufacturing Technology Management, 2005. 
[23] J. Machuca, M. S. Díaz, and M. A. Gil, "Adopting and implementing advanced manufacturing technology: new data on key factors from the aeronautical industry," International Journal of Production Research, vol. 42, pp. 3183-3202, 2004.

[24] J. Fortune and D. White, "Framing of project critical success factors by a systems model," International journal of project management, vol. 24, pp. 53-65, 2006.

[25] R. Yusuff, S. Saberi, and N. Zulkifli, "A comparison on the capabilities of Malaysian SMEs with different equity structure in implementing advanced manufacturing technologies," Asian International Journal of Science and Technology in Production and Manufacturing Engineering, vol. 1, pp. 63-75, 2008.

[26] R. Kumar, H. Singh, and R. Chandel, "Exploring the key success factors of advanced manufacturing technology implementation in Indian manufacturing industry," Journal of Manufacturing Technology Management, 2018.

[27] I. Dey, Qualitative data analysis: A user friendly guide for social scientists: Routledge, 2003.

[28] S. G. da Costa and E. P. de Lima, "Advanced manufacturing technology adoption: an integrated approach," Journal of Manufacturing Technology Management, 2009.

[29] H. Sun and F. Gertsen, "Organizational changes related to advanced manufacturing technology in the production area," International Journal of Production Economics, vol. 41, pp. 369-375, 1995/10/01/ 1995.

[30] M. H. Small, "Justifying investment in advanced manufacturing technology: a portfolio analysis," Industrial Management \& Data Systems, 2006.

[31] R. Millen and A. S. Sohal, "Planning processes for advanced manufacturing technology by large American manufacturers," Technovation, vol. 18, pp. 741-750, 1998/10/01/ 1998.

[32] J. B. Song, D. S. Dai, and Y. Q. Song, "The relationship between change of organizational structure and implementation of advanced manufacturing technology: An empirical study," in Proceedings of 2006 International Conference on Management Science and Engineering, ICMSE'06 (13th), 2006, pp. 782-786.

[33] H. Sun, "Current and future patterns of using advanced manufacturing technologies," Technovation, vol. 20, pp. 631-641, 2000/11/01/2000.

[34] X. Sun, Y. Tian, and G. Cui, "The empirical study on the impact of advanced manufacturing technology on organizational structure and human resources management," in International Conference on Management Science and Engineering, 2007, pp. 57-69.

[35] K. K. Boyer, P. T. Ward, and G. K. Leong, "Approaches to the factory of the future: An empirical taxonomy," Journal of Operations Management, vol. 14, pp. 297-313, 1996.

[36] N. Y. G. Lai, K. H. Wong, D. Halim, J. Lu, and H. S. Kang, "Industry 4.0 Enhanced Lean Manufacturing," in 2019 8th International Conference on Industrial Technology and Management (ICITM), 2019, pp. 206-211.

[37] M. C. Ungan, "Manufacturing best practices: implementation success factors and performance," Journal of Manufacturing Technology Management, 2007.

[38] C. M. McDermott and G. N. Stock, "Organizational culture and advanced manufacturing technology implementation," Journal of Operations Management, vol. 17, pp. 521-533, 1999/08/01/ 1999.

[39] S. Khazanchi, M. W. Lewis, and K. K. Boyer, "Innovation-supportive culture: The impact of organizational values on process innovation," Journal of operations management, vol. 25, pp. 871-884, 2007.

[40] H. Paul and B. Suresh, "Manufacturing strategy through planning and control techniques of advanced manufacturing technology," International Journal of Technology Management, vol. 6, pp. 233-242, 1991.

[41] T. Koc and E. Bozdag, "The impact of AMT practices on firm performance in manufacturing SMEs," Robotics and Computer-Integrated Manufacturing, vol. 25, pp. 303-313, 2009.

[42] M. Frohlich, "How do you successfully adopt an advanced manufacturing technology?," European Management Journal, vol. 16, pp. 151-159, 1998. 
[43] A. M. Sanchez, "Adopting advanced manufacturing technologies: Experience from Spain," Journal of Manufacturing Systems, vol. 15, pp. 133-140, 1996/01/01/ 1996.

[44] R. K. Singh, S. K. Garg, S. Deshmukh, and M. Kumar, "Modelling of critical success factors for implementation of AMTs," Journal of Modelling in Management, 2007.

[45] J. Rahardjo and S. bin Yahya, "Advanced manufacturing technology implementation process in SME: critical success factors," Jurnal Teknik Industri, vol. 12, pp. pp. 101-108, 2010.

[46] H. Singh and R. Kumar, "Hybrid methodology for measuring the utilization of advanced manufacturing technologies using AHP and TOPSIS," Benchmarking: An International Journal, 2013.

[47] R. Young and E. Jordan, "Top management support: Mantra or necessity?," International journal of project management, vol. 26, pp. 713-725, 2008.

[48] R. Ahmed and N. Azmi bin Mohamad, "Exploring the relationship between multi-dimensional top management support and project success: An international study," Engineering Management Journal, vol. 28, pp. 54-67, 2016.

[49] S. Jafari, M. Osman, M. Rosnah, and S. Tang, "A consensus on critical success factors for enterprise resource planning systems implementation: the experience of Malaysian firms," International journal of manufacturing technology and management, vol. 17, pp. 396-407, 2009.

[50] C. K. Parsons, R. C. Liden, E. J. O'Connor, and D. H. Nagao, "Employee Responses to TechnologicallyDriven Change: The Implementation of Office Automation in a Service Organization," Human Relations, vol. 44, pp. 1331-1356, 1991/12/01 1991.

[51] J. S. Cook and L. L. Cook, "Achieving competitive advantages of advanced manufacturing technology," Benchmarking for Quality Management \& Technology, 1994.

[52] J.-m. Choe, "Impact of management accounting information and AMT on organizational performance," Journal of Information Technology, vol. 19, pp. 203-214, 2004.

[53] Y. E. Spanos and I. Voudouris, "Antecedents and trajectories of AMT adoption: the case of Greek manufacturing SMEs," Research Policy, vol. 38, pp. 144-155, 2009.

[54] N. E. Waldeck and Z. M. Leffakis, "HR perceptions and the provision of workforce training in an AMT environment: an empirical study," Omega, vol. 35, pp. 161-172, 2007.

[55] K. K. Boyer, G. K. Leong, P. T. Ward, and L. J. Krajewski, "Unlocking the potential of advanced manufacturing technologies," Journal of operations management, vol. 15, pp. 331-347, 1997.

[56] J. P. MacDuffie, "Human resource bundles and manufacturing performance: Organizational logic and flexible production systems in the world auto industry," ilr Review, vol. 48, pp. 197-221, 1995.

[57] D. Boothby, A. Dufour, and J. Tang, "Technology adoption, training and productivity performance," Research Policy, vol. 39, pp. 650-661, 2010.

[58] B. Barua and M. O. Islam, "Key Success Factors for Implementation of Advanced Manufacturing Technologies (AMTs)-Case Study Conducted on Selected Pharmaceutical Companies in Bangladesh," BUSECON, vol. 15, 2008.

[59] B. E. Patuwo and M. Y. Hu, "The human factor in advanced manufacturing technology adoption," International Journal of Operations \& Production Management, 1998.

[60] C. A. M. Monge, S. S. Rao, M. E. Gonzalez, and A. S. Sohal, "Performance measurement of AMT: a crossregional study," Benchmarking: An International Journal, vol. 13, pp. 135-146, 2006.

[61] A. Efstathiades, S. A. Tassou, G. Oxinos, and A. Antoniou, "Advanced manufacturing technology transfer and implementation in developing countries: The case of the Cypriot manufacturing industry," Technovation, vol. 20, pp. 93-102, 2000.

[62] J. R. Meredith, "Implementing the automated factory," Journal of Manufacturing Systems, vol. 6, pp. 1-13, 1987/01/01/ 1987. 
[63] R. Turner, A. Ledwith, and J. Kelly, "Project management in small to medium-sized enterprises," Management Decision, 2012.

[64] S. S. Ariss, T. Raghunathan, and A. Kunnathar, "Factors affecting the adoption of advanced manufacturing technology in small firms," SAM Advanced Management Journal, vol. 65, p. 14, 2000.

[65] C. Hofmann and S. Orr, "Advanced manufacturing technology adoption-the German experience," Technovation, vol. 25, pp. 711-724, 2005.

[66] M. Putterill, W. Maguire, and A. S. Sohal, "Advanced manufacturing technology investment: criteria for organizational choice and appraisal," Integrated Manufacturing Systems, 1996.

[67] M. H. Small and I. J. Chen, "Investment justification of advanced manufacturing technology: An empirical analysis," Journal of Engineering and Technology Management, vol. 12, pp. 27-55, 1995/07/01/ 1995.

[68] C. Yu, X. Xu, and Y. Lu, "Computer-Integrated Manufacturing, Cyber-Physical Systems and Cloud Manufacturing - Concepts and relationships," Manufacturing Letters, vol. 6, pp. 5-9, 2015.

[69] P. Leitão, A. W. Colombo, and S. Karnouskos, "Industrial automation based on cyber-physical systems technologies: Prototype implementations and challenges," Computers in Industry, vol. 81, pp. 11-25, 2016/09/01/ 2016.

[70] D. Jayasekara, K. Pawar, and S. Ratchev, "A Framework to Assess Readiness of Firms for Cloud Manufacturing," in 2019 IEEE International Conference on Engineering, Technology and Innovation (ICE/ITMC), 2019, pp. 1-12.

[71] S. Peters, "A readiness level model for new manufacturing technologies," Production Engineering, vol. 9, pp. 647-654, 2015.

[72] A. Abdollahzadegan, A. R. Che Hussin, M. Moshfegh Gohary, and M. Amini, "The Organizational Critical Success Factors for Adopting Cloud Computing in SMEs," Journal of Information Systems Research and Innovation (JISRI), vol. 4, pp. 67-74, 2013.

[73] R. Plant and L. Willcocks, "Critical Success Factors in International ERP Implementations: A Case Research Approach," Journal of Computer Information Systems, vol. 47, pp. 60-70, 2007/03/01 2007. 